\title{
Exploring the role of testosterone in the cerebellum link to neuroticism: From adolescence to early adulthood
}

\author{
Dennis J.L.G. Schutter ${ }^{1,}$, Rosa Meuwese ${ }^{2,3}$, Marieke G.N. Bos ${ }^{2,3}$, Eveline A. Crone ${ }^{2,3}$, Jiska S. Peper ${ }^{2,3}$ \\ ${ }^{1}$ Donders Institute for Brain, Cognition and Behaviour, Radboud University, Montessorilaan 3, 6525 HR \\ Nijmegen, The Netherlands \\ ${ }^{2}$ Institute of Psychology, Leiden University, Wassenaarseweg 52, 2333 AK Leiden, The Netherlands \\ ${ }^{3}$ Leiden Institute for Brain and Cognition, The Netherlands
}

Address correspondence to: Dennis J.L.G. Schutter, Donders Institute for Brain, Cognition and Behaviour, Radboud University, Montessorilaan 3, 6525 HR Nijmegen, The Netherlands, +31 2436 11027,d.schutter@donders.ru.nl 


\section{Abstract}

Previous research has found an association between a smaller cerebellar volume and higher levels of neuroticism. The steroid hormone testosterone reduces stress responses and the susceptibility to negative mood. Together with in vitro studies showing a positive effect of testosterone on cerebellar gray matter volumes, we set out to explore the role of testosterone in the relation between cerebellar gray matter and neuroticism. Structural magnetic resonance imaging scans were acquired, and indices of neurotic personality traits were assessed by administering the depression and anxiety scale of the revised NEO personality inventory and Grays's behavioural avoidance in one hundred and forty-nine healthy volunteers between 10 and 27 years of age. Results demonstrated an inverse relation between total brain corrected cerebellar volumes and neurotic personality traits in adolescents and young adults. In males, higher endogenous testosterone levels were associated with lower scores on neurotic personality traits and larger cerebellar gray matter volumes. No such relations were observed in the female participants which may be due to general differences in endogenous testosterone levels. Analysis showed that testosterone significantly mediated the relation between male cerebellar gray matter and measures of neuroticism. Our findings on the interrelations between endogenous testosterone, neuroticism and cerebellar morphology provide a cerebellum-oriented framework for the susceptibility to experience negative emotions and mood in adolescence and early adulthood.

Key words: Behavioural Inhibition; Cerebellum; Gray matter; Neuroticism; Testosterone 


\section{Introduction}

Neuroticism refers to the personality trait that is characterized by the tendency to experience negative thoughts and feelings associated with behavioural avoidant behaviour (Eysenk, 1967). An important feature of neuroticism is that individuals typically display lower thresholds for experiencing negative emotions in response to stressors and take more time to recover and regain internal bodily homeostasis (McEwen, 1998). It has been shown that neurotic individuals are inclined to show a systemic bias to behavioural avoidance and experience negative emotions even in the absence of stressors further add to the established clinical observation that neuroticism is a risk factor for mood disorders (Clark et al., 1994). Disturbances in internal regulatory functions are increasingly considered to play a leading role in the transition between normal and pathological mood states (Kalisch et al., 2014). In support of this view, research has found abnormal activity in several limbic and cortical brain areas, which are known for their involvement in the regulation of stress, autonomic activity, bodily rhythms and homeostasis (Pruessner et al., 2010). Among these areas the hypothalamus, amygdala and prefrontal cortex have probably received most attention in scientific research.

Another less well established brain region proposed to be part of the neural machinery dedicated to homeostatic regulation and mood is the cerebellum. Even though the idea of cerebellum contributions to emotion and mood was already postulated in the nineteen-seventies (for a review see Schmahmann, 1997), systematic research on the role of the cerebellum in emotion, mood and psychiatric illnesses has been relatively scarce (Schmahmann and Sherman, 1998; Schutter and Van Honk, 2005). However, increasing evidence confirms the proposed involvement of the cerebellum in the experience and regulation of emotions and mood states (Schutter, 2016). One line of evidence comes from clinical reports that describe difficulties in emotion and mood regulation of patients with neurcognitive deficits due to cerebellar damage that cannot be explained by the presence of neurological impairments (Schmahmann, 2010). Administration of disruptive transcranial magnetic stimulation to the cerebellum of healthy volunteers has shown to impair 
emotion regulation causing a negative mood state (Schutter and van Honk, 2009). In a more recent structural 3T magnetic resonance imaging (MRI) association study in adults, we found evidence for an association between cerebellar volumes and neuroticism in a non-clinical community sample (Schutter et al., 2012). More specifically, cerebellar volume corrected for total brain volume inversely related to the proneness to experience anxious and depressive states. Several metaanalyses of functional magnetic resonance imaging are in support of the structural MRI data (Stoodely and Schmahmann, 2009; Keren-Happuch et al., 2014; Van Overwalle et al., 2014). Cerebellum activation in the detection and evaluation of threat cues coincides with the idea that the cerebellum is part of a brain circuit dedicated to stress regulation (Han et al., 2008; Schutter, 2012). Especially, the idea that the cerebellum is part of the autonomic physiological response pattern of the brain to threat cues and controls stress responses relevant for goal-directed behavior has been demonstrated by more recent fMRI studies (Schraa-Tam et al., 2012; Eisenbarth et al., 2016).

To further understand the relation between cerebellar volume and neurotic personality traits, the monosynaptic reciprocal connections between the cerebellum and hypothalamus are particularly notable (Haines et al., 1997). By controlling the release of hormones, the hypothalamus is a critical brain structure responsible for the regulation of stress responses, autonomic activity, bodily homeostasis and mood (Haines et al., 1984, 1997; Kelts and Hoehn, 1978; Ramnani, 2006). In addition to the hypothalamus-pituitary-adrenal axis linked to the release of stress hormone cortisol, the hypothalamic-pituitary-gonadal axis also plays an important role in governing the release of sex steroids as part of the fight-flight reaction (Toufexis et al., 2014). The discovery of sex steroid receptor binding sites on the cerebellum offers an additional feedback mechanism by which the sex steroid testosterone is able to influence the cerebello-hypothalamic coupling. Recent empirical findings show that androgen receptors in the Purkinje (output) cells are modulated by endogenous testosterone levels in male rats and further underscore the link between the cerebellum and the neuro-endocrine system (Perez-Pouchoulen et al., 2016). Moreover, in vitro studies have established that testosterone reduces oxidative stress-induced cell death of cerebellar gray matter (Ahlbom et 
al., 1999; Tsutsui et al., 2013). The association between low levels of testosterone and smaller gray matter volume agrees with clinical findings involving male participants with Klinefelter syndrome (Bryant et al., 2011) and effects of testosterone deprivation therapy on gray matter volumes of patients with prostate cancer (Chao et al., 2013).

The presence of steroid hormone receptors in cerebellar tissue offers a physiological basis for steroid hormonal modulation of cerebellar functions. In addition to the cerebello-hypothalamic link, recent debates on the involvement of the cerebellum in cognition and emotion are inspired by existing neuro-anatomical connections. In particular, the cerebello-cortical loops provide a basis for contributions of the cerebellum to processes associated with motivational direction and cognitive control (Schutter and van Honk, 2005). This is further supported by evidence of a topographical map in the cerebellum of motor control on the one hand, and cognitive and affective processes on the other hand (Stoodely and Schmahmann, 2010). Because of its uniform anatomical microstructure, the cerebellum is well suited to process signals from all parts of the brain. This so-called universal cerebellar transform (UCT) function is argued to promote internal homeostasis by monitoring and regulating signals coming from the autonomic, limbic and cortical regions of the brain (Schmahmann, 2004). The UCT has its origins in the processing of multimodal inputs stemming from the existing cerebellar connections with cortical and limbic parts of the brain. In particular, with the direct reciprocal connections to the hypothalamus the cerebellum acts as a monitor of central and peripheral (bodily) information processing related to internal homeostasis. Disruption of internal homeostasis, that is a mismatch between central and bodily state, activates the UCT for coordinating internal neural processes to restore equilibrium. From this perspective, anxiety and depression are the experiential correlates of disrupted bodily homeostasis. Suboptimal UCT function or chronic overstimulation may contribute to difficulties in establishing and maintaining homeostasis leading to neurotic traits (Schutter, 2012, 2016).

On the phenomenological level, testosterone has anxiolytic and antidepressant properties as evidenced by animal and human research (Carrier et al., 2015; Giltay et al., 2012; Hofer et al., 2013). 
In agreement, experimentally reducing endogenous testosterone and oestrogen levels in healthy female adults reduced reward-related brain activity and increased self-reported depression ratings (Macoveanu et al., 2016). Although results do not provide conclusive evidence on the efficacy of testosterone treatment in mood disorders, some studies suggest that testosterone protects against the development and progression of mood disorders, and acts as a primer for subsequent pharmaceutical interventions (Carrier and Kabbaj, 2012; Frye and Walf, 2009). Furthermore, there is increasing evidence that the onset and development of mood disorders starts during adolescence (Zahn-Waxler et al., 2008). Adolescence is a developmental stage roughly between 10-18 years of age characterized by a sharp rise in sex steroid hormones. Adolescence is proposed to be a sensitive period for sex steroid-induced brain maturation (Cunningham et al., 2007; Koolschijn et al., 2014; Peper and Dahl, 2013; Peper et al., 2015). In adolescents, lower levels of testosterone have been associated with the presence of anxious and depressive symptoms (Granger et al., 2003, but see Duke et al., 2014). It has been suggested that sex steroid levels in combination with environmental stress and neurotic temperament constitute a vulnerability factor for the onset of mood disorders during adolescence (Mueller, et al., 2014; Silk et al., 2012; Zinbarg et al., 2016). The findings suggest that the associations between cerebellar volumes, neurotic personality traits and endogenous testosterone levels may already be present in the adolescent period. Finally, earlier research has shown a significant positive relation between gray matter volumes and testosterone in adolescent boys, but not in girls suggesting that testosterone may have a different effect on brain morphology in male as compared to female adolescents (for review see Peper et al., 2011).

To address these questions, we conducted an exploratory study to examine the interrelations between endogenous testosterone levels in larger sample size as previously reported by Schutter and colleagues (2012), cerebellar volumes and neuroticism in adolescent and adult healthy volunteers. Consistent with our previous findings and existing literature on the role of testosterone in structural brain anatomy and mood, we tested the following three hypotheses: (1) Cerebellar volumes are inversely correlated with neuroticism; (2) Endogenous testosterone levels 
are inversely correlated to neuroticism; (3) The association between cerebellar volume and neuroticism is mediated by endogenous testosterone levels.

\section{Method}

\subsection{Participants}

This study was part of a two-year follow-up scan in the longitudinal 'BrainTime' research project (e.g., Braams et al., 2016; Peters et al., 2016). Of the 254 participants that were enrolled in the follow-up, ninety one healthy adolescents $(q=46, \hat{\delta}=45)$ aged between 10 and 17 years and fifty eight adults $(q=30, \delta=28)$ aged between 18 and 27 years, mean age \pm SD, $17.4 \pm 3.3$ years, had complete data records and were included in the current study. Reasons for exclusion were (1) MRI scan could not be obtained due to braces or use of intrauterine device, (2) insufficient quality of the MRI due to moving, (3) missing saliva samples, (4) no reliable hormone assay due to polluted saliva, (5) questionnaires that were not filled out, and (6) no data on alcohol and cigarette use. Eighteen of the thirty-five volunteers that were examined in our original prior study by Schutter et al. (2012) volunteered to participate again two years later. None of the participants had a history of psychiatric or neurological conditions. History of alcohol consumption and smoking in the past month was inquired to control for the influence of alcohol, cannabis and tobacco smoking on cerebellar volumes (Schutter et al., 2012; Torvik and Torp, 1986). Volunteers were naïve to the hypotheses tested in this study and were paid for participation. Written informed consent was obtained from participants > 12 years and informed assent from participants $<18$ years. Informed consent was obtained from both parents when participants were younger than 18 years of age. This study was part of a large longitudinal project and was approved by the local ethical review board of Leiden University, Leiden, the Netherlands, and carried out in accordance with the standards set by the Declaration of Helsinki (Fortaleza Amendments).

\subsection{Measures of neuroticism}


Personality traits related to anxiety and depression were rated with the Dutch version of the two corresponding 8-item subscales of the revised NEO Personality Inventory (NEO-PI-R) (Hoekstra et al., 1996; Kendler et al., 2004). The subscale anxiety measures personality characteristics associated with the experience of nervousness, tension, fear and worry. The subscale depression measures personality characteristics relates to the vulnerability to experience feelings of guilt, sadness, hopelessness and loneliness. Responses were collected on a 5 point scale $(1=$ strongly disagree; 2 = moderately disagree; 3 = neither disagree nor agree; 4 = moderately agree; 5 = strongly agree). The subscale anxiety and depression are highly interdependent, $r=0.62, p<0.0001$, and to limit the number of statistical analyses a composite NEO score (cNEO) was computed. The cNEO indexes emotional instability and subjective feelings of inadequacy (Hoekstra et al., 1996), and is associated with an increased risk of developing mood disorders (Kendler et al., 2004).

To increase construct validity of self-reported neuroticism, the Carver and White's Behavioural Inhibition System (BIS) scale was administered to assess avoidance-related behaviour (Carver and White, 1994; Sutton and Davidson, 1997). The BIS scale is a validated self-report measure consisting of 7 items. Responses are collected on a 4 point scale $(1=$ strongly disagree; $2=$ moderately disagree; 3 = moderately agree; 4 = strongly agree). The BIS scale is positively correlated cNEO, $r=0.61, p<0.001$, as they share a sensitivity to negative and undesirable stimuli paralleled by punishment sensitivity and an innate behavioural tendency away from these stimuli (Elliot and Thrash, 2005).

\subsection{MRI acquisition and pre-processing}

Scanning was performed on a $3 T$ Achieva whole body scanner (Philips, Best, The Netherlands) at Leiden University Medical Centre. A high-resolution 3D T1-Fast Field Echo scan was obtained $\left(\mathrm{TR}=9.760 \mathrm{~ms} ; \mathrm{TE}=4.59 \mathrm{~ms}\right.$, flip angle $=8$ degrees, 140 slices, $0.875 \times 0.875 \times 1.2 \mathrm{~mm}^{3}$ voxels, FOV $=224 \times 168 \times 177 \mathrm{~mm}^{3}$ ) with a total scan duration of $296 \mathrm{~s}$. (Schutter et al., 2012). All T1 scans were reviewed and cleared by a radiologist. No anomalous findings were reported. MRI scans 
were individually checked on motion artefacts or other sources of signal loss. Total brain and gray and white matter volumes of the left and right cerebellum were measured automatically using the software FreeSurfer v5.3.0. (Dale et al., 1999; Fischl and Dale, 2010; Fischl et al., 1999). In brief, processing consisted of removal of non-brain tissue (Segonne et al., 2004), and automatic segmentation of (sub)cortical white and gray matter structures (Fischl et al., 2002). These procedures are detailed in great length in prior publications and on the FreeSurfer website (http://surfer.nmr.mgh.harvard.edu/).

\subsection{Endogenous testosterone levels}

Morning saliva samples were collected at home, directly after waking up on the day of MRscanning to limit the influence of circadian rhythms and intra-individual daily fluctuations (Dabbs et al., 1990). Participants were instructed not to eat or brush their teeth before collecting saliva. To control for hormonal fluctuations across the menstrual cycle, saliva samples of post-menarcheal girls were collected on the same day within the early follicular phase of the menstrual cycle (day 7) when oestradiol and progesterone are relatively low (Mihm et al., 2011), and ensures maximum comparison between post-menarche girls. Similarly, girls using oral contraceptives collected a saliva sample on the last day within their stopping period (day 7). Girls using contraceptives without a stopping period, such as hormonal intrauterine devices, were excluded from participating in this study. Saliva samples were assayed for endogenous testosterone levels at the Department of Clinical Chemistry of the Free University Medical Centre (VUMC), Amsterdam, and the Netherlands. Salivary testosterone level was determined by isotope dilution - online solid phase extraction liquid chromatography - tandem mass spectrometry (ID-XLC-MS/MS) (Peper et al., 2015a; Peper et al., 2015b; Büttler et al., 2016). ID-XLC-MS/MS is superior in specificity and lower variation of hormonal levels as compared to radioimmunoassay (RIA) (Büttler et al., 2016) as LC-MS/MS can separate highly similar molecules, such as androstenedione from testosterone, and is able to reliably measure low levels of testosterone ( $<10 \mathrm{pmol} / \mathrm{l})$ (Handelsman and Wartofsky 2013; Owen et al., 2016). 
Testosterone concentrations in saliva samples were analyzed in duplicate. Measurements of samples of which the duplicate measurement had a difference $>15 \%$ were repeated. The intra-assay coefficient of variation describes the variation of results within a data set obtained from one experiment and is expressed by the intra-assay coefficient of variation (intra-assay CV) to monitor deviations within the same assay. Each sample is measured several times, and then \% CV is calculated for each sample by dividing the standard deviation (SD) of a set of measurements by the set mean, and multiplying by 100 . Finally, the average of the individual CVs is denoted as intra assay $\mathrm{CV}$. The inter-assay variation describes the variation of results obtained from repeated experiments and expressed by inter-assay coefficient of variation (inter-assay CV) to monitor the precision of results between different assays. Concentration values in $\mathrm{pmol} / \mathrm{l}$ are derived from the molar weight

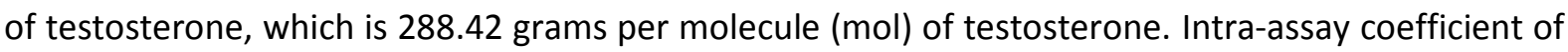
variation (CV) was $11 \%$ and $4 \%$, at 10 and 140 pmoL/L. Inter-assay CV was $8 \%$ and 5\%, at 31 and 195 pmol/L.

\subsection{Statistical analyses}

Partial correlation coefficients were computed on the whole sample between total, white and gray matter cerebellar volume corrected for total brain volume and the measure of neuroticism and behavioural avoidance controlling for age, alcohol consumption in the past month, cigarette smoking in the past month and cannabis use in the past month (Schutter et al., 2012). If a relation was significant, follow-up analyses were performed to further delineate contributions of the left and right cerebellar hemisphere. Next, the associations were examined separately for adolescents and young adults.

Effects of endogenous testosterone levels on cerebellar volume and measures of neuroticism were examined separately for males and female participants (for a rationale see Peper et al., 2013; Peper et al., 2015; Peters et al., 2015). Bootstrapped mediation analysis was performed to investigate whether endogenous levels of testosterone mediate the anticipated relation between 
cerebellar volume and measures of neuroticism (Preacher and Hayes, 2004, 2008). Bootstrapping is a nonparametric resampling procedure, which is an additional method advocated for testing mediation that does require the normality assumption of the sampling distribution (Preacher and Hayes, 2008). The bootstrapping method involves recurrent sampling from the data set and estimating the indirect effect in each resampled data set (for an authoritative review of methodology and procedure see Preacher and Hayes, 2008). Raw data was non-parametrically resampled $(n=1000)$ to estimate the confidence intervals $(\mathrm{Cl})$ of the indirect effect of endogenous testosterone levels corrected for age, alcohol, smoking, and cannabis use in past month. The mediation analysis was repeated with switched roles of testosterone and cerebellar volume to explore the specificity of endogenous testosterone levels as a mediator. The alpha level of significance was set at $<0.05$ (two-tailed).

\section{Results}

Descriptives of the main variables of the study are depicted in Table 1.

Table 1. Means and standard deviations of the main study variables.

$<<$ Insert Table 1 about here $\gg>$

\subsection{Cerebellar volumes and measures of neuroticism}

$$
<<\text { Insert Figure } 1 \text { about here >> }
$$

For our primary analysis the hypothesized inverse relation between total cerebellar volume

(Figure 1) and cNEO score was significant, $r=-0.25, p=0.003$ (Figure 2A). Follow-up secondary correlations demonstrated that the relation between cerebellar volume and cNEO score was 
explained by the contribution of cerebellar gray matter, $r=-0.31, p<0.001$ (Figure 2B), whereas the contribution of white matter was not significant, $r=0.06, p=0.447$ (Figure 2C). Left and right cerebellar gray matter volume contributed equally to the observed relation between cerebellar gray matter volume and cNEO score, $Z=1.03, p=0.152$. The cNEO score neither correlated to total brain volume, $r=-0.08, p=0.360$, nor total gray matter volume, $r=-0.12, p=0.156$, supporting a specific effect on cerebellum gray matter.

$<<$ Insert Figure 2A-C about here $>>$

Separate analyses for the adolescent and young adult subject groups yielded significant partial correlations between cerebellar gray matter volume and cNEO score, $r=-0.25, p=0.021$ in the adolescents, as well as in the young adults, $r=-0.37, p=0.006$. The associations between cerebellar white matter volume and cNEO score did not reach statistical significance in the adolescents, $r=$ $0.067, \mathrm{p}=0.536$, and young adults, $r=-0.12, p=0.407$. The correlation value between gray matter volume and cNEO scores explained a similar amount of variation between the adolescent and adult subject group, $Z=1.14, p=0.13$. Left and right cerebellar gray matter volume contributed equally to the observed relation between cerebellar gray matter volume and cNEO score in the adolescent, $Z=$ $0.77, p=0.221$, as well as in the young adult subject group, $Z=0.05, p=0.480$. Additionally, we tested whether the non-significant differences between the adolescent and young adult group resulted from regressing out the contribution of age in the relation between cerebellum volume and neuroticism. Running ANOVAs with adolescent versus young adult groups between subject factor did not result in differences in levels of neuroticism and total cerebellar volumes when controlling only for smoking, alcohol and cannabis use ( $p$-values $>0.266)$. Although gray and white matter were different between adolescents and young adults (both $p$-values $<0.001$ ), within group correlations between measures neuroticism and cerebellar volumes were not significant ( $p$-values $>0.13$ ). In sum, these control tests do not suggest that the lack of a group differences was driven by age. 
Additional support for the validity on the relation between cerebellar morphology and neuroticism was provided by the correlations between cerebellar volumes and BIS scores. BIS score was inversely related to total cerebellar volume, $r=-0.30, p<0.001$ (Figure 3A). The relation between total cerebellar volume and BIS score could be explained by gray, $r=-0.35, p<0.001$, (Figure 3B) and not white matter cerebellar volume, $r=0.01, p=0.980$ (Figure 3C). Left and right cerebellar gray matter contributed equally to the observed relation, $Z=0.36, p=0.359$. In line with the findings on the cNEO score, BIS score neither correlated to total brain volume, $r=-0.11, p=0.20$, nor total gray matter volume, $r=-0.14, p=0.089$.

$<<$ Insert Figure 3A-C about here $>>$

Separate analyses for the adolescent subject group yielded a significant correlation between cerebellar gray matter volume and BIS score, $r=-0.26, p=0.014$, while the association between cerebellar white matter volume and BIS score did not reach statistical significance, $r=0.20, p=0.07$. In the young adult subject group, the relation between cerebellar gray matter volume and BIS score was significant, $r=-0.33, p=0.014$. No correlation was found between cerebellum white matter and BIS score, $r=-0.02, p=0.892$. Left and right cerebellar gray matter volume contributed equally to the observed relation between cerebellar gray matter volume and BIS score in the adolescent, $Z=$ $0.24, p=0.444$, as well as in the young adult subject group, $Z=0.49, p=0.312$.

\subsection{Endogenous testosterone, cerebellar gray matter volumes and measures of neuroticism}

Endogenous testosterone levels in female participants did not show any correlations with cerebellar volume or self-reported questionnaires, $p^{\prime} s>0.29$. In the male participant group, endogenous testosterone levels significantly correlated with cNEO, $r=-0.34, p=0.005$ (Figure 4A), and BIS scores, $r=-0.45, p<0.001$ (Figure 4B). Planned follow-up analyses showed that, in the adolescent group ( $\mathrm{n}=72$ ), the correlations between testosterone and cNEO, $r=-0.40, p=0.007$, and 
BIS score, $r=-0.50, p=0.001$, were significant. In contrast, the correlations between testosterone and cNEO, $r=-0.28, p=0.145$, and BIS score, $r=-0.19, p=0.34$, in the male adult group $(n=28)$ did not reach significance. Thus, the group correlations seem to be predominantly driven by the adolescent participants, although the correlations between the adolescent and adult group were not statistically different (both Z-values $\leq 1$ ).

$<<$ Insert Figure 4A/B about here $>>$

Male endogenous testosterone levels were positively associated with total cerebellum volume, $r=0.29, p=0.017$. This correlation could be explained by the relation between gray matter volume of the right cerebellar hemisphere, $r=0.34, p=0.005$ (Figure 5). Inspection of the scatterplot showed that the data distribution was left-skewed. Removal of extreme values that exceeded three times the interquartile range $(n=3)$ did not significantly affect the relationship, $r=$ 0.299, $p=0.013$. Also, a non-parametric Spearman rank-order correlation in which the sampling distribution can be obtained without requiring the distribution probability of two variables and is less influenced by extreme values was significant, $r h o=0.340, p=0.003$. These additional analyses suggest that the association is not explained by the left-skewed distribution. No correlation was found for the left cerebellar hemisphere, $r=0.04, p=0.738$. Also, no relations were observed in the female participant group, all $p^{\prime} s>0.415$.

\footnotetext{
$<<$ Insert Figure 5 about here $>>$
}

\subsection{Mediation analysis}

Bootstrap mediation analysis showed that testosterone significantly mediated the relation between right cerebellar gray volume and cNEO score in the male participant group, $95 \% \mathrm{Cl}=-4.15-$ - 0.25 (Figure 6A), meaning that in males with a relatively high level of $T$, the association between 
the cerebellum and cNEO scores is suppressed. A second bootstrap mediation analyses showed that testosterone also significantly mediated the relation between right cerebellar gray volume and BIS score in the male participant group, $95 \% \mathrm{Cl}=-0.26--0.04$ (Figure 6B). No differences were observed between adolescents and young adults. The mediation analysis for examining the specificity of testosterone as a mediator showed that right cerebellar gray matter did neither significantly mediate the relation between testosterone and cNEO, $95 \% \mathrm{Cl}=-5.52-0.19$, nor the relation between testosterone and $\mathrm{BIS}, 95 \% \mathrm{Cl}=-0.21-0.04$.

$$
<<\text { Insert Figure 6A/B about here }>>
$$

\section{Discussion}

The aim of this study was to investigate the relation between cerebellar volume, measures of neuroticism and endogenous testosterone levels in a non-clinical sample of adolescents and young adults.

Results show that the association between larger cerebellar volumes and lower levels of neuroticism remains present in a fourfold sample size - compared to our previous study restricted to adults only (Schutter et al., 2012). Here, we show that gray and not white matter volumes account for the earlier reported relation between cerebellar volume and neurotic personality traits. Furthermore, we found associations between cerebellar volumes and BIS scores which provide complementary support that the cerebellum is associated with punishment sensitivity and cognitive biases towards negative and undesirable stimuli. Our findings fit results of a recent systematic review describing the relation between the cerebellar morphology and personality characteristics (Petrosini et al., 2016).

In addition, we explored the hypothesis that the relation between cerebellar volume and neuroticism may already be present during adolescence. In line with this hypothesis, we found 
significant associations between cerebellar total and gray matter volumes and the neurotic personality traits in the adolescent participants group. The inverse relation between cerebellar volumes and neurotic personality traits in adolescents and adults concurs with findings of reduced cerebellum activity and smaller gray matter volumes and higher levels of harm avoidance ( $O^{\prime}$ Gorman et al., 2006). Indeed, harm avoidance shows considerable overlap with behavioural avoidance and neurotic personality characteristics including high punishment sensitivity, worrying, lack of initiative, shyness and anxiety (Cloninger et al., 1993; De Fruyt et al., 2000). Functional neuroimaging studies have demonstrated neural activity of the cerebellum correlates with error monitoring and related to probabilistic inference modelling and context updating (Ide and $\mathrm{Li}, 2011$ ). The cerebellum is proposed to be involved in routines to minimize uncertainty and maximize predictability. The associations between the cerebellum and neuroticism may be indicative for a role of the UCT in the tendency to worry, anxiety and subjective feelings of loss of control (Schutter, 2016). The fact that neurotic personality traits contribute to the prevalence of mood disorders is supported by several anatomical studies showing that patients with mood disorders have significantly smaller cerebellar gray matter volumes (Adamaszek et al., 2016; Lai and Wu, 2015; Peng et al., 2011). Conversely, increased blood flow through cerebellar tissue and higher levels of coherent activity in the cerebellum have been found to positively correlate to novelty seeking, positive affect and approachrelated behaviour (O'Gorman et al., 2006; Wei et al., 2011). The latter features are typical of an extraverted personality style and linked to higher endogenous levels of testosterone (Eysenk, 1967; Smeets-Janssen et al., 2015). Extraversion is negatively correlated with symptoms of depression and anxiety (Jylhä and Isometsä, 2006), and the risk of mood disorders is significantly higher in individuals who score low on extraversion and high on neuroticism (Grav et al., 2012). Taken together, our present observations hint at the possibility that cerebellar volumes in adolescents may express an early anatomical vulnerability factor to mood disorders.

Although it is difficult to establish whether the volumetric deviations from controls are part of the pathophysiology of mood disorders, growing empirical evidence suggests that this may well 
be the case. One line of such evidence comes from neuro-anatomical studies that have revealed the connections of the cerebellum with subcortical and cortical brain regions and most notably the monosynaptic projections to the hypothalamus which provides an important neuro-anatomical basis for the homeostatic regulation of mood (Schutter, 2012; Schutter, 2016). Interestingly, testosterone is among the primary end-products of the HPG- axis and plays an important role in motivation and mood. Several administration studies have demonstrated that testosterone has anxiolytic and antidepressants effects in healthy volunteers (Enter et al., 2014; Herman et al., 2006; Van Honk et al., 2003). The idea that mood improvements associated with androgen administration in hypogonadal men is attributed to higher levels of testosterone (Ebinger et al., 2009) strengthens the idea that testosterone plays a role in the relationship between cerebellar volumes and neuroticism. In further support of this idea, our analyses show that testosterone mediates the association between cerebellar gray matter and neurotic personality traits. This mediation effect was observed for the right cerebellar hemisphere in the male participants group. When we performed the analysis separately for adolescence and young adults the mediation effect disappeared in both groups. Statistical power issues as a result of sample size may explain the absence of finding on the subgroup level. Lastly, even though our control tests do not suggest that the lack of a group differences was driven by age, the unequal distribution of age within the subgroups may have introduced statistical difficulties and should therefore be considered a limitation of the study.

On a final note, endogenous circulating testosterone levels start to decrease from adulthood onwards and reaches a hypogonadal level in a significant proportion of males over 60 years of age (Harman et al., 2001). Together with psychological data indicating that neuroticism can increase in old age (Steunenberg et al., 2005), our findings suggest that older hypogonadal men scoring high on neuroticism may have reduced gray matter volume of the right cerebellum. In sum, although we could not show that the relation between cerebellar volumes and neuroticism was present in the adolescent and young adult group separately, other studies suggest that more research is needed in this area. 
It should furthermore be acknowledged that our study does not allow making strong inferences on the direction of the correlations. However, based on the established protective effects of testosterone on cerebellar morphology and mood, we speculate that higher testosterone levels reduce neuroticism through its positive effect on right cerebellar gray matter in males. Some additional support for this idea may come from results of an ad hoc bootstrap mediation analysis we performed in the male subjects group. In this analysis, right cerebellar gray matter was entered as the mediator variable to examine its effect on the relation between testosterone levels and neurotic personality traits. Results showed that right cerebellar gray matter did not significantly mediate the relation between testosterone levels and CNEO, and the relation between testosterone and BIS score.

Our findings were restricted to the right hemisphere only, and even though the literature is scarce on cerebellar lateralization in emotion, mood and personality, there is some evidence for distinct involvement of the right cerebellum in emotion processing (Tan et al., 2015). These findings concur with functional magnetic resonance imaging (fMRI) data showing distinct cerebellar activation in the processing of primary emotions (Baumann and Mattingley, 2012). Specifically, activation of the right cerebellar hemisphere seems associated with the processing of anger and fear. According to these authors, the right cerebellum is part of the neural circuit implicated in threat processing and mediates autonomic and endocrine responses to fear and anger (Baumann and Mattingley, 2012). At least for males, this idea fits the proposed role of cerebello-hypothalamic interactions between testosterone, neurotic personality traits and mood regulation (Schutter 2016).

The observation that the relation between right cerebellar gray matter and testosterone was confined to male participants sample concurs with animal research showing antidepressant effects of testosterone administration in socially isolated male, but not in female rats (Carrier and Kabbaj, 2012). Another explanation could be that in women, other sex steroids play a role in affecting the relation between cerebellum and neuroticism. For example, the female sex hormone oestradiol has, analogous to testosterone in men, positive effects on cerebellar morphology and mood in women. 
Specifically, the binding of oestradiol to androgen receptors has been thought to produce both functional and organizational effects on the cerebellum (Haraguchi et al., 2012; Napolitano et al., 2014). The discovery that oestradiol has protective effects on the cerebellum (Hedges et al., 2012; Sasahara et al., 2007) raises the possibility that hormonal effects on neuroticism and cerebellar gray matter in women are mediated through oestradiol (Koibuchi and Ikeda 2013). Synthesis of oestradiol and testosterone depends on progesterone which has shown to promote dendritic growth, spinogenesis, and synaptogenesis of Purkinje cells (Dean et al., 2008; Tsutsui, 2013). Recent empirical findings show that androgen receptors in the Purkinje cells are also directly modulated by endogenous testosterone levels in male rats (Perez-Pouchoulen et al., 2016). Since oestradiol is aromatized from testosterone, oestradiol may play a more pronounced role in cerebellar gray matter volumes of women than testosterone.

Variation in steroidogenic enzymatic activity may contribute to differences between the sexes as well. A methodology oriented explanation for the null findings in women may relate to their low endogenous testosterone levels found as compared to men. Variation needed to detect reliable individual differences on the group level may have been too limited. This may especially be the case when one also considers the intra-assay coefficient of variation in determining testosterone levels. Repeated assessments of hormonal levels during the course of a day, a week or even longer periods will increase measurement sensitivity and offer the possibility to study hormonal rhythms and fluctuations over time.

Next to hormones, genetic make-up likely contribute to the current associations as well. Cerebellar gray matter volumes have been linked to $\mathrm{X}$ chromosome dosage independent of the organizational effects of foetal testosterone (Lentini et al., 2013). Women that lack a normal second X chromosome show an overall increase of cerebellar volume as compared to female controls (Cutter et al., 2006). Voxel based morphometry (VBM) analyses indicate that in particular enlarged larger gray matter volume of the cerebellar hemispheres accounts for the larger total cerebellar volume (Cutter et al., 2006). In addition to genetic contributions, cross-sex hormone administration 
in transsexuals shows that manipulation of sex steroids causes organizational changes in brain morphology (Hulshoff-Pol et al., 2006). Together, results of these studies suggest a role of both genetics and steroid hormones in cerebellar volumes for explaining intra- and inter-individual differences. How exactly genetics contribute and interact with non-genetic factors with respect to the presently observed relations with neuroticism remains unclear and warrants further research.

Moreover, oestradiol is aromatized from testosterone, so the androgeneric effects of testosterone in men may, at least in part, be established by oestradiol in women. Variation in steroidogenic enzymatic activity is also likely to contribute to differences between the sexes. A methodology oriented explanation for the null findings in women may relate to their low endogenous testosterone levels found as compared to men. Because statistical mean and variance are positively correlated, the inter-individual variation for detecting reliable individual differences on the group level may have been too limited. This may especially be the case when one also considers the intra-assay coefficient of variation in determining testosterone levels. Repeated assessments of hormonal levels during the course of a day, a week or even longer periods will increase measurement sensitivity and offer the possibility to study hormonal rhythms and fluctuations over time.

In addition, even though mediation analyses provide insights into the effects of other variables (e.g., testosterone levels) on a particular relation (e.g., cerebellar volume and neuroticism), this form of analyses remains correlational of nature. Mediation analyses are employed to understand the nature of a relationship by examining the mechanisms by which one variable influences a second variable through an intervening (mediator) variable (Hayes, 2009). It should be noted that even though mediation is a causal phenomenon, mediation analyses do not offer a way of establishing genuine causality (Hayes and Preacher, 2014). Mediation analysis is a statistical approach that is able to determine whether a relation between variables exists and what the magnitude of this relation is. This may contribute to the likelihood of causality, but certainly does not prove it (Hayes and Preacher, 2014). Correlations and mediation analyses may nonetheless 
provide the first necessary step along the process of establishing causation by demonstrating a relationship between the variables of interest. The next logical step would be to conduct hormonal and behavioural manipulation studies to establish the direction of the relation. Based on the existing literature on the anatomical and behavioural effects of testosterone administration in animals and humans, we speculate that testosterone contributes to the relation between cerebellar morphology and neuroticism in males.

As for our statistical analyses, we adopted a hypothesis driven hierarchical testing approach. The series of planned correlations started with reproducing our previously reported findings in a large sample (Schutter et al., 2012). Significant correlations were followed by subsequent correlations to get more insights into the nature of the correlations between cerebellar volume and neuroticism. Adding the BIS scale to the study was to provide further support construct validity of the psychological concept of neuroticism. In our opinion, this complementary approach further adds to the reliability of the present results on the interrelation between neuroticism, cerebellum and testosterone. Moreover, due of inherent differences in bio-availability of testosterone between the sexes, correlations involving testosterone were performed separately for male and female volunteers. The sample size and age range allowed us to explore the relations in adolescence and young adulthood. Although the strength of the correlations is modest, our findings are comparable to results of similar research that have investigated the relations between brain volumes, selfreports and hormones (Koolschijn et al., 2014; Wei et al., 2015). Restrictions in the range of values of the variables of interest, type of study design and quality of the measurement are sources of error variance that lower the correlation coefficient. Other factors that we could not control for, such as the role of genetics and effects of other hormones, may have further lowered the signal-to-noise ratio.

Finally, we evaluated alcohol consumption, cigarette smoking and cannabis use in the past month to control for the effects of substance use on cerebellum volumes (Schutter et al., 2012). It should however be noted that we also questioned participants about lifetime alcohol consumption, 
smoking and cannabis use. The correlations between reported lifetime and last month for alcohol consumption, $r=0.890, p<0.001$, smoking, $r=0.923, p<0.001$, and cannabis use, $r=0.551, p<$ 0.001 , were moderate-to-high. Because accuracy is typically higher for the recollection of recent events and lifetime reports are likely to be biased by recent events (Menon, 1993), we only included the reports of the last month in our partial correlations. Still, in spite of the statistical controls, effects of substance use on cerebellum volumes cannot be excluded.

In sum, even though we found statistically significant relations our results should be interpreted with caution and the association between cerebellar gray matter and testosterone seen as preliminary.

\section{Conclusion}

This is, to our knowledge, the first study that has successfully integrated predictions from both the animal and human literature to demonstrate associations between cerebellar morphology, testosterone and neuroticism. Our findings provide an empirical basis for establishing the direction of the correlations in future work. The interrelations between testosterone, behavioural indices of emotional vulnerability and cerebellar morphology provide a cerebellum oriented framework for hormone-brain interactions in the susceptibility to experience normal and pathological forms of negative mood. Our findings indicate that these relations are not exclusively limited to adults, but can already be observed in the adolescent population. The results offer a theoretical and empirical starting point for further testing the idea that testosterone may influence cerebellar volumes and act as a neuro-protective agent against the susceptibility to worry, experience context inappropriate anxiety and hypersensitivity to punishment in males. Replication and additional studies to address the functional mechanisms and behaviours underlying the association between testosterone and the cerebellum are warranted. 
Competing financial interests: Author declares no competing financial interests.

Acknowledgements: This work was supported by NWO Innovational research grants 452-07-012 (D.S.) and 451-10-007 (J.P.) and an ERC Starting Grant 2010-StG-263234 (E.C.). The authors thank dr. P.C. Koolschijn for making the cerebellum reconstruction figure. 


\section{References}

Adamaszek, M., D'Agata, F., Ferrucci, R., Habas, C., Keulen, S., Kirkby, K.C., Leggio, M., Mariën, P., Molinari, M., Moulton, E., Orsi, L., Van Overwalle, F., Papadelis, C., Priori, A., Sacchetti, B., Schutter, D.J., Styliadis, C., Verhoeven, J., 2016. Consensus Paper: Cerebellum and Emotion. Cerebellum Aug 2. [Epub ahead of print].

Ahlbom, E., Grandison, L., Bonfoco, E., Zhivotovsky, B., Ceccatelli, S., 1999. Androgen treatment of neonatal rats decreases susceptibility of cerebellar granule neurons to oxidative stress in vitro. Eur. J. Neurosci. 11, 1285-1291.

Baumann, O., Mattingley, J.B., 2012. Functional topography of primary emotion processing in the human cerebellum. Neuroimage 61, 805-811.

Braams, B.R., Peper, J.S., Van der Heide, D., Peters, S., Crone, E.A., 2016. Nucleus accumbens response to rewards and testosterone levels are related to alcohol use in adolescents and young adults. Dev. Cogn. Neurosci. 17, 83-93.

Bryant, D.M., Hoeft, F., Lai, S., Lackey, J., Roeltgen, D., Ross, J., Reiss, A.L., 2011. Neuroanatomical phenotype of Klinefelter syndrome in childhood: a voxel-based morphometry study. J. Neurosci. 31, 6654-6660.

Büttler, R.M., Peper, J.S., Crone, E.A., Lentjes, E.G., Blankenstein, M.A., Heijboer, A.C., 2016. Reference values for salivary testosterone in adolescent boys and girls determined using isotope-dilution liquid-chromatography tandem mass spectrometry (ID-LC-MS/MS). Clin. Chim. Acta 456, 15-18.

Carrier, N., Kabbaj, M., 2012. Testosterone and imipramine have antidepressant effects in socially isolated male but not female rats. Horm. Behav. 61, 678-685.

Carrier, N., Saland, S.K., Duclot, F., He, H., Mercer, R., Kabbaj, M., 2015. The anxiolytic and antidepressant-like effects of testosterone and estrogen in gonadectomized male rats. Biol. Psychiatry 78, 259-269. 
Carver, C.S., White, T.L., 1994. Behavioral inhibition, behavioral activation, and affective responses to impending reward and punishment: The BIS/BAS scales. J. Pers. Soc. Psychol. 67, 319-333.

Chao, H.H., Hu, S., Ide, J.S., Uchio, E., Zhang, S., Rose, M., Concato, J., Li, C.S., 2013. Effects of androgen deprivation on cerebral morphometry in prostate cancer patients--an exploratory study. PLoS One 8, e72032.

Clark, L.A., Watson, D., Mineka, S., 1994. Temperament, personality, and the mood and anxiety disorders. J. Abnorm. Psychol. 103, 103-116.

Cloninger, C.R., Svrakic, D.M., Przybeck, T.R., 1993. A psychobiological model of temperament and character. Arch. Gen. Psychiatry 50, 975-990.

Costa, P.T., McCrae, R.R., 1992. Revised NEO personality inventory and NEO five-factor inventory professional manual. Lutz, FL: Psychological Assessment Resources Inc.

Cunningham, R.L., Claiborne, B.J., McGinnis, M.Y., 2007. Pubertal exposure to anabolic androgenic steroids increases spine densities on neurons in the limbic system of male rats. Neuroscience 150, 609-615.

Dabbs, J.M., 1990. Salivary testosterone measurements: Reliability across hours, days, and weeks. Physiol. Behav. 48, 83-86.

Dale, A.M., Fischl, B., Sereno, M.I., 1999. Cortical surface-based analysis. I. Segmentation and surface reconstruction. Neuroimage 9,179-194.

De Fruyt, F., Van de Wiele, I., Van Heeringen, C., 2000. Cloninger's psychobiological model of temperament and character and the five-factor model of personality. Pers. Indiv. Diff. 29, 441-452.

Dean, S.L., McCarthy, M.M., 2008. Steroids, sex and the cerebellar cortex: implications for human disease. Cerebellum 7, 38-47.

Duke, S.A., Balzer, B.W., Steinbeck, K.S., 2014. Testosterone and its effects on human male adolescent mood and behavior: A systematic review. J. Adolesc. Health. 55, 315-322. 
Eisenbarth, H., Chang, L.J., Wager, T.D., 2016. Multivariate brain prediction of heart rate and skin conductance responses to social threat. J. Neurosci. 36, 11987-11998.

Elliot, A.J., Thrash, T.M., 2001. Approach and avoidance motivation in personality: Approach and avoidance temperaments and goals. J. Pers. Soc. Psychol. 82, 804-818.

Enter, D., Spinhoven, P., Roelofs, K., 2014. Alleviating social avoidance: effects of single dose testosterone administration on approach-avoidance action. Horm. Behav. 65, 351-354.

Eysenck, H.J., 1967. The Biological Basis of Personality. Springfield: Charles C. Thomas.

Fischl, B., Dale, A.M., 2000. Measuring the thickness of the human cerebral cortex from magnetic resonance images. Proc. Natl. Acad. Sci. U. S. A. 97, 11050-11055.

Fischl, B., Salat, D.H., Busa, E., Albert, M., Dieterich, M., Haselgrove, C., van der Kouwe, A., Killiany, R., Kennedy, D., Klaveness, S., Montillo, A., Makris, N., Rosen, B., Dale, A.M., 2002. Whole brain segmentation: Automated labeling of neuroanatomical structures in the human brain. Neuron 33, 341-355.

Fischl, B., Sereno, M.I., Dale, A.M., 1999. Cortical surface-based analysis. II: Inflation, flattening, and a surface-based coordinate system. Neuroimage 9, 195-207.

Frye, C.A., Walf, A.A., 2009. Depression-like behavior of aged male and female mice is ameliorated with administration of testosterone or its metabolites. Physiol. Behav. 97, 266-269.

Giltay, E.J., Enter, D., Zitman, F.G., Penninx, B.W., van Pelt, J., Spinhoven, P., Roelofs, K., 2012. Salivary testosterone: associations with depression, anxiety disorders, and antidepressant use in a large cohort study. J. Psychosom. Res. 72, 205-213.

Granger, D.A., Shirtcliff, E.A., Zahn-Waxler, C., Usher, B., Klimes-Dougan, B., Hastings, P., 2003. Salivary testosterone diurnal variation and psychopathology in adolescent males and females: Individual differences and developmental effects. Dev. Psychopathol. 15, 431-449.

Grav, S., Stordal, E., Romild, U.K., Hellzen, O., 2012. The relationship among neuroticism, extraversion, and depression in the HUNT Study: in relation to age and gender. Issues Ment. Health Nurs. 33, 777-785. 
Haines, D.E., Dietrichs, E., Sowa, T.E., 1984. Hypothalamo-cerebellar and cerebello-hypothalamic pathways: a review and hypothesis concerning cerebellar circuits which may influence autonomic centers affective behavior. Brain Behav. Evol. 24, 198-220.

Haines, D.E., Dietrichs, E., Mihailoff, G.A., McDonald, E.F., 1997. The cerebellar-hypothalamic axis: basic circuits and clinical observations. Int. Rev. Neurobiol. 41, 83-107.

Han, S., Gao, X., Humphreys, G.W., Ge, J., 2008. Neural processing of threat cues in social environments. Hum. Brain Mapp. 29, 945-957.

Handelsman, D.J., Wartofsky, L., 2013. Requirement for mass spectrometry sex steroid assays in the journal of clinical endocrinology and metabolism. J. Clin. Endocrinol. Metab. 98, 3971-3973. Haraguchi, S., Sasahara, K., Shikimi, H., Honda, S., Harada, N., Tsutsui, K., 2012. Estradiol promotes Purkinje dendritic growth, spinogenesis, and synaptogenesis during neonatal life by inducing the expression of BDNF. Cerebellum 11, 416-417.

Harman, S.M., Metter, E.J., Tobin, J.D., Pearson, J., Blackman, M.R., 2001. Baltimore Longitudinal Study of Aging. Longitudinal effects of aging on serum total and free testosterone levels in healthy men. J. Clin. Endocrinol. Metab. 86, 724-731.

Hayes, A.F., 2009. Beyond Baron and Kenny: Statistical mediation analyses in the new millennium. Commun. Monogr. 76, 408-420.

Hayes, A.F., Preacher, K.J., 2014. Statistical mediation analysis with a multicategorical independent variable. Br. J. Math. Stat. Psychol. 67, 451-470.

Heath, R.G., Harper, J.W., 1974. Ascending projections of the cerebellar fastigial nucleus to the hippocampus, amygdala, and other temporal lobe sites: evoked potential and histological studies in monkeys and cats. Exp. Neurol. 45, 268-287.

Hedges, V.L., Ebner, T.J., Meisel, R.L., Mermelstein, P.G., 2012. The cerebellum as a target for estrogen action. Front. Neuroendocrinol. 33, 403-411.

Hermans, E.J., Putman, P., Baas, J.M., Koppeschaar, H.P., Van Honk, J., 2006. A single administration of testosterone reduces fear-potentiated startle in humans. Biol. Psychiatry 59, 872-874. 
Hoekstra, H.A., Ormel, J., De Fruyt, F., 1996. Handleiding NEO persoonlijkheidsvragenlijsten: NEO-PIR en NEO-FFI. Lisse: Swets Test Services.

Höfer, P., Lanzenberger, R., Kasper, S., 2013. Testosterone in the brain: neuroimaging findings and the potential role for neuropsychopharmacology. Eur. Neuropsychopharmacol. 23, 79-88.

Hulshoff-Pol, H.E., Cohen-Kettenis, P.T., Van Haren, N.E., Peper, J.S., Brans, R.G., Cahn, W., Schnack, H.G., Gooren, L.J., Kahn, R.S.. 2006. Changing your sex changes your brain: influences of testosterone and estrogen on adult human brain structure. Eur. J. Endocrinol. 155, S107S114.

Ide, J.S., Li, C.S., 2011. A cerebellar thalamic cortical circuit for error-related cognitive control. Neuroimage 54, 455-464.

Jylhä, P., Isometsä, E., 2006. The relationship of neuroticism and extraversion to symptoms of anxiety and depression in the general population. Depress. Anxiety 23, 281-289.

Kalisch, R., Müller, M.B., Tüscher, O., 2014. A conceptual framework for the neurobiological study of resilience. Behav. Brain Sci. 38, e128.

Kelts, K.A., Hoehn, M.M., 1978. Hypothalamic atrophy. J. Clin. Psychiatry. 39, 363-365.

Kendler, K.S., Kuhn, J., Prescott, C.A., 2004. The interrelationship of neuroticism, sex, and stressful life events in the prediction of episodes of major depression. Am. J. Psychiatry 161, 631-636.

Keren-Happuch, E., Chen, S.H., Ho, M.H., Desmond, J.E., 2014. A meta-analysis of cerebellar contributions to higher cognition from PET and fMRI studies. Hum. Brain Mapp. 35, 593-615.

Koibuchi, N., Ikeda, Y., 2013. Hormones and cerebellar development. In: Manto, M., Gruol, D.L., Schmahmann, J.D., Koibuchi, N., Rossi, F., editors. Handbook of the cerebellum and cerebellar disorders. Dordrecht: Springer. p 319-339.

Koolschijn, P.C., Peper, J.S., Crone, E.A., 2014. The influence of sex steroids on structural brain maturation in adolescence. PLoS One 9, e83929.

Lai, C.H., Wu, Y.T., 2015. The gray matter alterations in major depressive disorder and panic disorder: Putative differences in the pathogenesis. J. Affect. Disord. 186, 1-6. 
Lentini, E., Kasahara, M., Arver, S., Savic, I., 2013. Sex differences in the human brain and the impact McEwen, B.S., 1998. Stress, adaptation, and disease: allostasis and allostatic load. Ann. N.Y. Acad. Sci. 840, 33-44.

Menon, G,. 1993. The effects of accessibility of information in memory on judgments of behavioral frequencies. J. Consum. Res. 20, 431-440.

Mihm, M., Gangooly, S., Muttukrishna, S., 2011. The normal menstrual cycle in women. Anim. Reprod. Sci. 124, 229-236.

Mueller, S.C., Grissom, E.M., Dohanich, G.P., 2014. Assessing gonadal hormone contributions to affective psychopathologies across humans and animal models. Psychoneuroendocrinology $46,114-128$.

Napolitano, M., Costa, L., Piacentini, R., Grassi, C., Lanzone, A., Gulino, A., 2014. 17ß-estradiol protects cerebellar granule cells against $\beta$-amyloid-induced toxicity via the apoptotic mitochondrial pathway. Neurosci. Lett. 561, 134-139.

O’Gorman, R.L., Kumari, V., Williams, S.C., Zelaya, F.O., Connor, S.E., Alsop, D.C., Gray, JA., 2006. Personality factors correlate with regional cerebral perfusion. Neuroimage 31, 489-495.

Owen, L.J., Wu, F.C., Büttler, R.M., Keevil, B.G., 2016. A direct assay for the routine measurement of testosterone, androstenedione, dihydrotestosterone and dehydroepiandrosterone by liquid chromatography tandem mass spectrometry. Ann. Clin. Biochem. 53, 580-587.

Peng, J., Liu, J., Nie, B., Li, Y., Shan, B., Wang, G., Li, K., 2011. Cerebral and cerebellar gray matter reduction in first-episode patients with major depressive disorder: A voxel-based morphometry study. Eur. J. Radiol. 80, 395-399.

Peper, J.S., Hulshoff -Pol, H.E, Crone, E.A., Van Honk, J., 2011. Sex steroids and brain structure in pubertal boys and girls: a mini-review of neuroimaging studies. Neuroscience 191, 28-37.

Peper, J.S., Dahl, R.E., 2013. Surging hormones: Brain-behavior interactions during puberty. Curr. Dir. Psychol. Sci. 22, 134-139. 
Peper, J.S., De Reus, M.A., Van den Heuvel, M.P., Schutter, D.J., 2015. Short fused? Associations between white matter connections, sex steroids, and aggression across adolescence. Hum. Brain Mapp. 36, 1043-1052.

Peper, J.S., Koolschijn, P.C., Crone, E.A., 2013. Development of risk taking: Contributions from adolescent testosterone and the orbito-frontal cortex. J. Cogn. Neurosci. 25, 2141-2150.

Peters, S., Jolles, D.J., Van Duijvenvoorde, A.C., Crone, E.A., Peper, J.S., 2015. The link between testosterone and amygdala-orbitofrontal cortex connectivity in adolescent alcohol use. Psychoneuroendocrinology 53, 117-126.

Peters, S., Van Duijvenvoorde, A.C., Koolschijn, P.C., Crone, E.A., 2016. Longitudinal development of frontoparietal activity during feedback learning: Contributions of age, performance, working memory and cortical thickness. Dev. Cogn. Neurosci. 19, 211-222.

Petrosini, L., Cutuli, D., Picerni, E., Laricchiuta, D., 2016. Viewing the personality traits through a cerebellar lens: A focus on the constructs of novelty seeking, harm avoidance, and alexithymia. Cerebellum, Jan 6.

Pruessner, J.C., Dedovic, K., Pruessner, M., Lord, C., Buss, C., Collins, L., Dagher, A., Lupien, S.J., 2010. Stress regulation in the central nervous system: evidence from structural and functional neuroimaging studies in human populations Psychoneuroendocrinology 35, 179191.

Ramnani, N., 2006. The primate cortico-cerebellar system: anatomy and function. Nat. Rev. Neurosci. 7, 511-522.

Sasahara, K.,Shikimi, H., Haraguchi, S., Sakamoto, H., Honda, S., Harada, N.,Tsutsui, K., 2007. Mode of action and functional significance of estrogen-inducing dendritic growth, spinogenesis, and synaptogenesis in the developing Purkinje cell. J. Neurosci. 27, 7408-7417.

Schmahmann, J.D., 1997. Rediscovery of an early concept. Int. Rev. Neurobiol. 41, 3-27.

Schmahmann, J.D., Sherman, J.C., 1998. The cerebellar cognitive affective syndrome. Brain 121, 561579. 
Schmahmann, J.D., 2004. Disorders of the cerebellum: ataxia, dysmetria of thought, and the cerebellar cognitive affective syndrome. J. Neuropsychiatry Clin. Neurosci. 16, 367-378.

Schmahmann, J.D., 2010. The role of the cerebellum in cognition and emotion: personal reflections since 1982 on the dysmetria of thought hypothesis, and its historical evolution from theory to therapy. Neuropsychol. Rev. 20, 236-260.

Schraa-Tam, C.K., Rietdijk, W.J, Verbeke, W.J., Dietvorst, R.C., Van den Berg, W.E., Bagozzi, R.P., De Zeeuw, C.I., 2012. fMRI activities in the emotional cerebellum: A preference for negative stimuli and goal-directed behavior. Cerebellum 11, 233-245.

Schutter, D.J., Van Honk, J., 2005. The cerebellum on the rise in human emotion. Cerebellum 4, 290 294.

Schutter, D.J., Van Honk, J., 2009. The cerebellum in emotion regulation: a repetitive transcranial magnetic stimulation study. Cerebellum 8, 28-34.

Schutter, D.J., 2012. The cerebello-hypothalamic-pituitary-adrenal axis dysregulation hypothesis in depressive disorder. Med. Hypotheses 79, 779-783.

Schutter, D.J., Koolschijn, P.C., Peper, J.S., Crone, E.A., 2012. The cerebellum link to neuroticism: a volumetric MRI association study in healthy volunteers. PLoS One 7, e37252.

Schutter, D.J., 2016. A cerebellar framework for predictive coding and homeostatic regulation in depressive disorder. Cerebellum 15, 30-33.

Segonne, F., Dale, A.M., Busa, E., Glessner, M., Salat, D., Fischl, B., 2004. A hybrid approach to the skull stripping problem in MRI. Neuroimage 22, 1060-1075.

Smeets-Janssen, M.M., Roelofs, K., Van Pelt, J., Spinhoven, P., Zitman, F.G., Penninx, B.W., Giltay, E.J., 2015. Salivary testosterone is consistently and positively associated with extraversion: Results from the Netherlands study of depression and anxiety. Neuropsychobiology 71, 7684.

Steunenberg, B., Twisk, J.W., Beekman, A.T., Deeg, D.J., Kerkhof, A.J., 2005. Stability and change of neuroticism in aging. J. Gerontol. B Psychol. Sci. Soc. Sci. 60, 27-33. 
Stoodley, C.J., Schmahmann, J.D., 2009. Functional topography in the human cerebellum: A metaanalysis of neuroimaging studies. Neuroimage 44, 489-501.

Stoodley, C.J., Schmahmann, J.D., 2010. Evidence for topographic organization in the cerebellum of motor control versus cognitive and affective processing. Cortex $46,831-844$.

Sutton, S.K., Davidson, R.J., 1997. Prefrontal brain asymmetry: A biological substrate of the behavioral approach and inhibition systems. Psychol. Sci. 8, 204-210.

Tan, R.H., Devenney, E., Kiernan, M.C., Halliday, G.M., Hodges, J.R., Hornberger, M., 2015. Terra incognita-cerebellar contributions to neuropsychiatric and cognitive dysfunction in behavioral variant frontotemporal dementia. Front. Aging Neurosci. 7, 121.

Torvik, A., Torp, S., 1986. The prevalence of alcoholic cerebellar atrophy: A morphometric and histological study of an autopsy material. J. Neurol. Sci. 75, 43-51.

Toufexis, D., Rivarola, M.A., Lara, H., Viau, V., 2014. Stress and the reproductive axis. J. Neuroendocrinol. 26, 573-586.

Tsutsui, K., Haraguchi, S., Hatori, M., Hirota, T., Fukada, Y., 2013. Biosynthesis and biological actions of pineal neurosteroids in domestic birds. Neuroendocrinology 98, 97-105.

Van Honk, J., Peper, J.S., Schutter, D.J., 2005. Testosterone reduces unconscious fear but not consciously experienced anxiety: Implications for the disorders of fear and anxiety. Biol. Psychiatry 58, 218-225.

Van Overwalle, F., Baetens, K., Mariën, P., Vandekerckhove, M., 2015. Social cognition and the cerebellum: A meta-analysis of over 350 fMRI studies. Neuroimage $86,554-572$.

Wang, M., 2011. Neurosteroids and GABA-A receptor function. Front. Endocrinol. (Lausanne) 2, 44. Wei, D., Du, X., Li, W., Chen, Q., Li, H., Hao, X., Zhang, L., Hitchman, G., Zhang, Q., Qiu, J., 2015. Regional gray matter volume and anxiety-related traits interact to predict somatic complaints in a non-clinical sample. Soc. Cogn. Affect. Neurosci. 10, 122-128. 
Wei, L., Duan, X., Yang, Y., Liao, W., Gao, Q., Ding, J.R., Zhang, Z., Zeng, W., Li, Y., Lu, G., Chen, H., 2011. The synchronization of spontaneous BOLD activity predicts extraversion and neuroticism. Brain Res. 1419, 68-75.

Zahn-Waxler, C., Shirtcliff, E.A., Marceau, K., 2008. Disorders of childhood and adolescence: gender and psychopathology. Annu. Rev. Clin. Psychol. 4, 275-303.

Zinbarg, R.E., Mineka, S., Bobova, L., Craske, M.G., Vrshek-Schallhorn, S., Griffith, J.W., WolitzkyTaylor, K., Waters, A.M., Sumner, J.A., Anand, D., 2016. Testing a hierarchical model of neuroticism and its cognitive facets: Latent structure and prospective prediction of first onsets of anxiety and unipolar mood disorders during 3 years in late adolescence. Clin. Psychol. Sci. doi: 10.1177/2167702615618162. 
Table 1. Means and standard deviations of the main study variables.

\begin{tabular}{|c|c|c|c|c|c|}
\hline & & $\begin{array}{l}\text { Adolescents } \\
(n=91)\end{array}$ & $\begin{array}{l}\text { Young adults } \\
(n=58)\end{array}$ & ANOVA & $\begin{array}{l}\text { Weighted total } \\
(n=149)\end{array}$ \\
\hline \multirow[t]{9}{*}{ Cerebellum volume (in cc) } & Gray & & & & \\
\hline & Left lobule & $45.27 \pm 5.53$ & $44.08 \pm 4.77$ & $F=1.82, p=0.180$ & $44.81 \pm 5.26$ \\
\hline & Right lobule & $45.93 \pm 5.52$ & $44.39 \pm 4.08$ & $F=2.96, p=0.088$ & $45.33 \pm 5.35$ \\
\hline & Total & $91.20 \pm 10.52$ & $88.47 \pm 9.06$ & $F=2.64, p=0.106$ & $90.14 \pm 10.04$ \\
\hline & White & & & & \\
\hline & Left lobule & $16.03 \pm 1.89$ & $16.34 \pm 1.66$ & $F=1.05, p=0.308$ & $16.15 \pm 1.81$ \\
\hline & Right lobule & $15.61 \pm 2.14$ & $16.09 \pm 1.72$ & $F=2.03, p=0.156$ & $15.80 \pm 2.00$ \\
\hline & Total & $31.64 \pm 3.67$ & $32.43 \pm 3.04$ & $F=1.85, p=0.175$ & $31.95 \pm 3.45$ \\
\hline & Grand total & $122.84 \pm 12.41$ & $120.90 \pm 10.50$ & $F=0.97, p=0.326$ & $122.09 \pm 11.70$ \\
\hline \multirow[t]{2}{*}{ Testosterone level (in picomol/L) } & Male & $261.22 \pm 149.82$ & $368.57 \pm 168.36$ & $F=8.06, p=0.006$ & $302.4 \pm 164.65$ \\
\hline & Female & $25.11 \pm 13.56$ & $27.67 \pm 16.79$ & $F=0.53, p=0.467$ & $26.11 \pm 14.87$ \\
\hline cNEO (range: 0-96) & & $41.96 \pm 9.37$ & $44.74 \pm 11.81$ & $F=2.55, p=0.113$ & $43.04 \pm 10.44$ \\
\hline BIS (range: 1-4) & & $2.74 \pm 0.46$ & $2.86 \pm 0.53$ & $F=2.12, p=0.148$ & $2.78 \pm 0.49$ \\
\hline
\end{tabular}


Figure 1. Illustration of a volumetric cerebellum reconstruction. 
Figure 2. Scatter plots with regression lines and individual $95 \%$ confidence interval depicting the whole group associations between cNEO score and total (A) nad gray matter volume of the cerebellum (B). 
Figure 3. Scatter plots with regression lines and individual $95 \%$ confidence interval depicting whole group associations between BIS score and total (A) and gray matter volume of the cerebellum (B). 
Figure 4. Scatter plot with regression lines and individual $95 \%$ confidence interval showing that endogenous testosterone is inversely related to CNEO (A) and BIS scores in the male subjects group (B). 
Figure 5. Positive association with regression line and individual $95 \%$ confidence interval between endogenous testosterone levels and gray matter volume of the right cerebellum in the male subject group. 
Figure 6. Endogenous testosterone levels in male participants significantly influences the relation between right cerebellar gray matter volume and neurotic personality traits. Higher levels of testosterone are associated with larger right cerebellar gray matter volumes and lower CNEO $(\mathbf{A})$ and BIS scores in males (B). 